\title{
Thermodynamic Functions for Fermions with Gravostatic and Electrostatic Interactions*
}

\author{
Peter Hertel ${ }^{\star \star}$, Heide Narnhofer, and Walter Thirring \\ Institute for Theoretical Physics, University of Vienna, Vienna, Austria
}

Received June 12, 1972

\begin{abstract}
The energy as function of entropy and the free energy as function of temperature is calculated rigorously for nonrelativistic fermions with$$
\frac{e^{\prime} e^{\prime \prime}-\kappa M^{\prime} M^{\prime \prime}}{\left|\vec{x}^{\prime}-\vec{x}^{\prime \prime}\right|}
$$

interactions. It is shown that in the appropriate thermodynamic limit the corresponding Thomas-Fermi equation becomes exact.
\end{abstract}

\section{Introduction}

In this paper we evaluate the thermodynamic functions for matter in the limit of many particles. By "matter" we mean nonrelativistic fermions interacting with Coulomb- and Newton potentials. For definiteness we consider two species of equally many fermions (electrons and protons, say) with masses $M_{1}, M_{2}$ and opposite charges $\pm e$. The reason why the thermodynamic functions can be calculated is that for a large number $N$ of particle pairs the system is compressed by gravitation to such an extent that the Thomas-Fermi equation becomes exact. The present results generalize those of a previous paper [1] in two respects:

a) Electrostatic forces are also included. Our results show that for $N \rightarrow \infty$ they cannot prevent the compression of the system. This is in accordance with the folklore that for

$$
N>\left(\frac{e^{2}}{\kappa M_{p}^{2}}\right)^{3 / 2} \sim 10^{54}
$$

atoms are squashed and one obtains a high-density plasma. For the ground state this fact has been demonstrated by Levy-Leblond [2].

b) We also show that if one calculates the energy as function of entropy in the microcanonical ensemble the relevant Thomas-Fermi

* Work supported by „Fonds zur Förderung der wissenschaftlichen Forschung“, Projekt Nr. 1391.

$\star \star$ On leave of absence from the University of Heidelberg, Germany. 
equation becomes exact. This result had been anticipated when a numerical calculation of these quantities in the purely gravitational case was carried out [3]. There it turned out that the phase transition which occurs in the canonical ensemble corresponds to a region of negative heat capacity in the microcanonical ensemble. This phenomenon has been found for models of stars [4] and of hadrons [5] and it is encountered for the good old $1 / r$-potential as well.

For those values of the energy where the heat capacity is positive (a Legendre transformation from entropy as a function of energy to the free energy as a function of temperature can then be defined) the microcanonical and the canonical ensemble give the same thermodynamical functions. There is, however, a region where the two ensembles are not equivalent.

More specifically, we study the limits

and

$$
\lim _{\substack{N \rightarrow \infty \\ N-1 \log \Omega \rightarrow s \\ N^{1 / 3} L \rightarrow l}} N^{-7 / 3} \frac{1}{\Omega} \sum_{v=1}^{\Omega} E_{v}(N, L) \equiv \Sigma(s, l)
$$

where $E_{v}(N, L)$ denotes the $v$-th eigenvalue - in ascending order - of a Hamiltonian describing the $2 N$ interacting particles which are enclosed in a cubic box of length $L$. The powers of $N$ in the above expressions are the same as in the purely gravitational case and are dictated by the following considerations: For noninteracting nonrelativistic fermions one has a one-parameter family of equivalent limits $N \rightarrow \infty$ with $N^{-\gamma} L$, $N^{2 \gamma-5 / 3} \bar{E}, N^{2 / 3-2 \gamma} \beta^{\prime}$, and $N^{-1} \log \Omega$ approaching constant values $l, \Sigma, \beta$, and $s$ respectively. Since in our case the ground state energy $E_{1}(N)$ $=\lim _{L \rightarrow \infty} E_{1}(N, L)$ goes like $N^{7 / 3}[2]$ we have to choose $\gamma=-\frac{1}{3}$ to assure $\lim _{l \rightarrow \infty} \lim _{s \rightarrow 0} \Sigma(s, l)=\lim _{N \rightarrow \infty} N^{-7 / 3} E_{1}(N)$. This law of corresponding states can also be inferred from the Thomas-Fermi equation with $1 / r$-interaction.

In this paper we shall demonstrate only that the (temperature dependent) Thomas-Fermi equation becomes exact, for a discussion of its numerical solution we refer to an earlier paper [3].

In our proof we cast into exact mathematical bounds the usual saying which goes along with the Thomas-Fermi equation: Since the singularity of the $1 / r$-potential prevents its uniform approximation by step functions we use our previous result to show that it can be removed without changing the thermodynamical functions significantly. We then subdivide the cube into sufficiently many small cubes and approximate the now continuous potential by another which is constant as long as a 
particle remains within a small cube. This will change the thermodynamical functions by a negligible amount only. Next the small cubes are separated by infinitely strong $\delta$-function barriers. Here the method of Ref. [1] turns out to be too cumbersome ${ }^{1}$, we adopt an idea of Griffiths and Robinson [6] instead: to each state of the system without walls a state of the system with walls is associated such that the expectation value of the Hamiltonian is only slightly larger. Now the problem is reduced to studying noninteracting fermions within boxes having an additional potential energy which depends on occupation numbers of boxes only. One has to find the distribution of particles among boxes which minimizes the mean or free energy for fixed entropy or temperature - this is equivalent to solving the Thomas-Fermi equation. Our final result is that the thermodynamical functions are, in the appropriate limit, those of noninteracting particles which move in a selfconsistently determined external potential.

Instead for a spherical vessel - as in Ref. [1] and [3] - we prove the above assertions for a cube. It is, however, evident that they are correct for any vessel form which can be approximated by cubes.

\section{Formulation of the Problem}

We want to investigate the statistical behaviour of two species $(\alpha=1,2)$ of equally many fermions of opposite charge $\left(e_{1}=e, e_{2}=-e\right)$ and with possibly different masses $M_{\alpha}\left(M=M_{1} \geqq M_{2}\right.$, say) which interact by gravitational and electrostatic forces. The $2 N$ particles are confined to move within a cubic box $\Lambda_{L} \equiv\left\{\vec{x} \in R^{3}: 0<x_{r}<L(r=1,2,3)\right\}$. This means that the Hilbert space $\mathscr{H}_{N, L}$ under discussion is made of (the equivalence classes of square-integrable, complex-valued functions $\psi\left(\vec{x}_{11}, \ldots, \vec{x}_{1 N}, \vec{x}_{21}, \ldots, \vec{x}_{2 N}\right)$ which are antisymmetric in the first $N$ variables $\vec{x}_{1 i}$, antisymmetric in the remaining variables $\vec{x}_{2 i}$ and have support in $\bar{\Lambda}_{L} \times \bar{\Lambda}_{L} \times \cdots \times \bar{\Lambda}_{L} \subset R^{6 N}$. Denote by $\mathscr{G}_{N . L} \subset \mathscr{H}_{N, L}$ the set of absolutely continuous functions, vanishing at the boundary of $\Lambda_{L} \times \Lambda_{L} \times \cdots \times \Lambda_{L}$ and having partial derivatives in $\mathscr{H}_{N, L}$, and by $\vec{\nabla}_{\alpha i}$ the gradient with respect to the $\alpha i$-th variable. We define the following sesqui-linear form $\mathscr{G}_{N, L} \times \mathscr{G}_{N, L} \rightarrow C$ :

$$
\begin{aligned}
(\Phi|H| \psi) \equiv \sum_{\alpha i} & \frac{1}{2 M_{\alpha}} \int d^{6 N} \boldsymbol{x}\left(\vec{\nabla}_{\alpha i} \Phi(x)\right)^{*}\left(\vec{\nabla}_{\alpha i} \psi(x)\right) \\
& +\frac{1}{2} \sum_{\substack{\alpha i \\
\beta k}}^{\prime} \int d^{6 N} \boldsymbol{x} \Phi^{*}(\boldsymbol{x}) \frac{e_{\alpha} e_{\beta}-\kappa M_{\alpha} M_{\beta}}{\left|\vec{x}_{\alpha i}-\vec{x}_{\beta k}\right|} \psi(\boldsymbol{x}) .
\end{aligned}
$$

${ }^{1}$ Indeed, Eq. (4.12) of Ref. [1] is an oversimplification and is valid if the radial contributions to the free energy are taken into account only. Including the angular quantum numbers, although trivial, leads to very messy formulae. 
$\boldsymbol{x} \in R^{6 N}$ stands for $\left(\vec{x}_{11}, \ldots, \vec{x}_{2 N}\right)$ and $\Sigma^{\prime}$ means that terms with $(\alpha, i)=(\beta, k)$ are to be discarded. The form is hermitian and bounded from below [2], i.e. there exists a number $b$ such that $(\Phi, \Phi)_{H} \equiv(\Phi|H+b| \Phi) \geqq(\Phi \mid \Phi)$ holds for $\Phi \in \mathscr{G}_{N, L}$. With this $\|\cdot\|_{H}$-norm $\mathscr{G}_{N, L}$ is a Hilbert space which means that it is also complete. It is $\|\cdot\|$-dense in $\mathscr{H}_{N, L}$.

The theorem of Riesz says that to any $\Phi \in \mathscr{H}_{N, L}$ corresponds a $\psi \in \mathscr{G}_{N, L}$ such that $(\Phi, \chi)=(\psi, \chi)_{H}$ holds for all $\chi \in \mathscr{G}_{N, L}$. One easily verifies that $\Phi \rightarrow \psi$, considered as a mapping $\mathscr{H}_{N, L} \rightarrow \mathscr{H}_{N, L}$ is linear, injective, hermitian, bounded and has range $\mathscr{D}_{N, L}$ which is dense in $\mathscr{G}_{N, L}$ in the $\|\cdot\|_{H}$-norm and consequently dense in $\mathscr{H}_{N, L}$ in the usual norm. Therefore its inverse is self-adjoint on the domain $\mathscr{D}_{N, L}$ and is equal to $b+$ the Hamiltonian $\hat{H}$ of the system under discussion, which is formally given by

$$
\hat{H}=-\sum_{\alpha i} \frac{\Delta_{\alpha i}}{2 M_{\alpha}}+\frac{1}{2} \sum_{\substack{\alpha i \\ \beta k}}^{\prime} \frac{e_{\alpha} e_{\beta}-\kappa M_{\alpha} M_{\beta}}{\left|\vec{x}_{i \alpha}-\vec{x}_{k \beta}\right|} .
$$

$\hat{H}$ has a discrete spectrum only, we label the eigenvalues in ascending order by $E_{v}\left(N, L, e^{2}, \kappa\right)$. It plays a secondary role only and we will never have to specify its domain $\mathscr{D}_{N, L}$ explicitly. The reason is that the mean energy $\bar{E}$ and the free energy $F$ can be obtained from the form $H$ :

and

$$
\bar{E}\left(N, \Omega, L, e^{2}, \kappa\right) \equiv \Omega^{-1} \inf _{\mathscr{\mathscr { F }} \Omega} \sum_{\Phi \in \mathscr{F}_{\Omega}}(\Phi|H| \Phi)
$$

$$
F\left(N, \beta, L, e^{2}, \kappa\right) \equiv-\beta^{-1} \log \sup _{\mathscr{F}} \sum_{\Phi \in \mathscr{F}} e^{-\beta(\Phi|H| \Phi)} .
$$

$\mathscr{F}_{\Omega}(\mathscr{F})$ is a set of $\Omega$ (finitely many) mutually orthogonal and normalized vectors of $\mathscr{G}_{N, L}$. Since $\mathscr{D}_{N, L}$ is dense in $\mathscr{G}_{N, L}$ in the $\|\cdot\|_{H}$-norm and $(\Phi|H| \Phi)$ is per definition continuous in this topology the infimum or supremum in (2.3), (2.4) can be restricted to all $\mathscr{F} \subset \mathscr{D}_{N, L}$ without changing the result. Then, by standard results, there is a relation with the eigenvalues of $\hat{H}$ :

$$
\bar{E}\left(N, \Omega, L, e^{2}, \kappa\right)=\Omega^{-1} \sum_{v=1}^{\Omega} E_{v}\left(N, L, e^{2}, \kappa\right)
$$

is the mean value of the first $\Omega$ eigenvalues of $\hat{H}$ and

is the usual free energy.

$$
F\left(N, \beta, L, e^{2}, \kappa\right)=-\beta^{-1} \log \sum_{\nu=1}^{\infty} e^{-\beta E_{\nu}\left(N, L, e^{2}, \kappa\right)}
$$

The following statement is a consequence of the behaviour of the kinetic and potential energy with respect to dilatations $x \rightarrow N^{\gamma} x$ :

$$
\Sigma(N, \Omega, l)=N^{2 \gamma-5 / 3} \bar{E}\left(N, \Omega, N^{\gamma} l, N^{-1 / 3-\gamma} e^{2}, N^{-1 / 3-\gamma} \kappa\right)
$$


and

$$
\Phi(N, \beta, l)=N^{2 \gamma-5 / 3} F\left(N, N^{-2 / 3+2 \gamma} \beta, N^{\gamma} l, N^{-1 / 3-\gamma} e^{2}, N^{-1 / 3-\gamma} \kappa\right)
$$

do not depend on $\gamma$.

Our aim is to calculate

$$
\Sigma(s, l)=\lim _{\substack{N \rightarrow x \\ N^{-1} \log \Omega \rightarrow s}} \Sigma(N, \Omega, l)
$$

and

$$
\Phi(\beta, l)=\lim _{N \rightarrow \infty} \Phi(N, \beta, l)
$$

for $s>0, l>0, \beta>0$.

\section{Substituting $1 / \mathrm{r}$ by a Regulated Function}

From now on we will employ the $N$-independent volume version of (2.7) and (2.8), i.e. choose $\gamma=0$.

$$
\left\{\begin{array}{c}
\hat{V}_{\mu} \\
\hat{U}_{\mu}
\end{array}\right\} \equiv \frac{1}{2} N^{-1} \sum_{\substack{\alpha i \\
\beta k}}^{\prime}\left\{\begin{array}{c}
e_{\alpha} e_{\beta}-\kappa M_{\alpha} M_{\beta} \\
e^{2}+\kappa M^{2}
\end{array}\right\} \frac{e^{-\frac{\mu}{l}\left|\vec{x}_{\alpha l}-\vec{x}_{\beta k}\right|}}{\left|\vec{x}_{\alpha i}-\vec{x}_{\beta k}\right|}
$$

are hermitian operators: $\mathscr{D}_{N, l} \rightarrow \mathscr{H}_{N, l}$. (Recall that $M$ is the larger of $M_{1}$ and $M_{2}$.) We also define the kinetic energy operator

$$
\hat{T} \equiv N^{-2 / 3} \sum_{\alpha i} \frac{-\Delta_{\alpha i}}{2 M_{\alpha}}: \mathscr{D}_{N, l} \rightarrow \mathscr{H}_{N, l}
$$

The region $\Lambda_{l}$ within which the particles are confined to move is now a cube of length $l$. We subdivide it into $g=h^{3}$ small cubes $\Lambda_{l / h}^{a}$ of equal length $l / h(a=1,2, \ldots, g)$. The centre of cube $\Lambda_{l / h}^{a}$ is denoted by $l \vec{\xi}_{a}$, its characteristic function is

$$
\theta_{a}(\vec{x}) \equiv\left\{\begin{array}{c}
1 \\
1 / 2 \\
1 / 4 \\
1 / 8 \\
0
\end{array}\right\} \text { if } \vec{x} \text { is }\left\{\begin{array}{l}
\text { inside } \\
\text { on the surface of } \\
\text { on an edge of } \\
\text { a corner of } \\
\text { outside }
\end{array}\right\} \overrightarrow{\Lambda_{l / h}^{a}}
$$

The operator for the number of particles of species $\alpha$ in cube $\Lambda_{l / h}^{a}$ is

$$
\hat{N}_{\alpha a} \equiv \sum_{i=1}^{N} \theta_{a}\left(\vec{x}_{\alpha i}\right)
$$


With the $\mu, g$-dependent $c$-numbers

$$
W_{\alpha a^{\prime} \beta b} \equiv\left\{\begin{array}{l}
\left(e_{\alpha} e_{\beta}-\kappa M_{\alpha} M_{\beta}\right) \frac{1-e^{-\mu\left|\vec{\xi}_{a}-\vec{\xi}_{b}\right|}}{\left|\vec{\xi}_{a}-\vec{\xi}_{b}\right| l} \\
\mu\left(e_{\alpha} e_{\beta}-\kappa M_{\alpha} M_{\beta}\right) / l
\end{array}\right\} \text { if }\left\{\begin{array}{c}
a \neq b \\
a=b
\end{array}\right\}
$$

we furthermore define

$$
\hat{V}_{\mu g} \equiv \frac{1}{2} N^{-1} \sum_{\substack{\alpha a \\ \beta b}} \hat{N}_{\alpha a} W_{\alpha a, \beta b} \hat{N}_{\beta b} .
$$

$\hat{V}_{\mu g}$ is an approximation - in the sense of inequalities (3.13) - to the original interaction $\hat{V}_{0} . \hat{T}, \hat{T}+\hat{V}_{0}, \hat{T}+\lambda \hat{U}_{0}, \hat{T}+\lambda \hat{U}_{\mu}$ and $\hat{T}+\hat{V}_{\mu g}$ are self-adjoint operators: $\mathscr{D}_{N, l} \rightarrow \mathscr{H}_{N, l}$ if $\mu>0$ and $\lambda \in R$. They correspond to sesqui-linear hermitian closed forms $T, T+V_{0}, T+\lambda U_{0}, T+\lambda U_{\mu}$ and $T+V_{\mu g}: \mathscr{G}_{N, l} \times \mathscr{G}_{N, l} \rightarrow C$ which are defined in analogy with (2.1).

Levy-Leblond's result [2] and Eq. (2.11) of Ref. [1], in the case of two species of identical fermions, read now:

$$
\begin{gathered}
0 \leqq U_{0} \leqq \lambda^{-1} T+\lambda a_{1} N ; \lambda \in R, a_{1}=2 M\left(e^{2}+\kappa M^{2}\right)^{2}, \\
0 \leqq U_{\mu} \leqq \mu^{-1 / 5}\left(T+a_{2} N\right) ; \mu>0, a_{2}=4 M^{3 / 2}\left(e^{2}+\kappa M^{2}\right)^{5 / 2} l^{1 / 2} .
\end{gathered}
$$

These bounds are valid for the operators as well since $\mathscr{D}_{N, l} \subset \mathscr{G}_{N, l}$.

For arbitrary $\varepsilon>0$ there is an integer $g_{0}(\varepsilon, \mu)$ such that

$$
\left|\sum_{a b} \theta_{a}\left(\vec{x}^{\prime}\right) \theta_{b}\left(\vec{x}^{\prime \prime}\right) \frac{1-e^{-\mu\left|\vec{\xi}_{a}-\vec{\xi}_{b}\right|}}{\left|\vec{\xi}_{a}-\vec{\xi}_{b}\right| l}-\frac{1-e^{-\frac{\mu}{l}\left|\vec{x}^{\prime}-\vec{x}^{\prime \prime}\right|}}{\left|\vec{x}^{\prime}-\vec{x}^{\prime \prime}\right|}\right| \leqq \frac{\varepsilon}{2}
$$

holds for $\vec{x}^{\prime}, \vec{x}^{\prime \prime} \in \Lambda_{l}$ and $g \geqq g_{0}(\varepsilon, \mu)$. Therefore

$$
V_{\mu q}-\bar{\varepsilon} N \leqq \frac{1}{2} N^{-1} \sum_{\substack{\alpha i \\ \beta k}}\left(e_{\alpha} e_{\beta}-\kappa M_{\alpha} M_{\beta}\right) \frac{1-e^{-\frac{\mu}{l}\left|\vec{x}_{\alpha i}-\vec{x}_{\beta k}\right|}}{\left|\vec{x}_{\alpha i}-\vec{x}_{\beta k}\right|} \leqq V_{\mu g}+\bar{\varepsilon} N
$$

and

$$
-U_{\mu}-\bar{\varepsilon} N+\mu a_{3} \leqq V_{\mu g}-V_{0} \leqq U_{\mu}+\bar{\varepsilon} N+\mu a_{3}
$$

with $a_{3}=\left(e^{2}+\frac{M_{1}^{2}+M_{2}^{2}}{2}\right) l^{-1}$ and $\bar{\varepsilon}=\varepsilon l^{-1}\left(e^{2}+\kappa M^{2}\right)$ results.

From (3.8), (3.11) and a consequence of (3.7):

$$
0 \leqq T \leqq T+\left(T-2 U_{0}\right)+4 a_{1} N \leqq 2\left(T+V_{0}\right)+4 a_{1} N
$$


we deduce the desired approximation:

$$
\frac{T+V_{\mu g}-c N}{1+2 \mu^{-1 / 5}} \leqq T+V_{0} \leqq \frac{T+V_{\mu g}+c N}{1-2 \mu^{-1 / 5}},
$$

where $c=\bar{\varepsilon}+N^{-1} \mu a_{3}+\mu^{-1 / 5} a_{2}+4 \mu^{-1 / 5} a_{1}$ can be made arbitrarily small by choosing $N, g$, and $\mu$ large enough.

We have to show next that such an approximation of forms (or Hamiltonian operators) is carried over to an approximation of the corresponding thermodynamical functions. Consider the mappings of hermitian sesqui-linear forms $A$ into $R$, defined by

$$
(A)_{\Omega}^{\text {m.c. }} \equiv \Omega^{-1} \inf _{\mathscr{F} \Omega} \sum_{\Phi \in \mathscr{F} \Omega}(\Phi|A| \Phi)
$$

and

$$
(A)_{\beta}^{\mathrm{c}} \equiv-\beta^{-1} \log \sup _{\mathscr{F}} \sum_{\Phi \in \mathscr{F}} e^{-\beta(\Phi|A| \Phi)},
$$

where $\mathscr{F}_{\Omega}(\widetilde{F})$ is a set of $\Omega$ (finitely many) orthonormalized vectors in the domain of $A$. (m.c. (c.) stand for microcanonical (canonical) average.) Both mappings are increasing:

$$
A \leqq B \quad \text { implies }(A) \leqq(B),
$$

$c$-number additive:

$$
(A+\lambda)=(A)+\lambda \text { for } \lambda \in R,
$$

and $(.)^{\text {m.c. }}$ is linear for $\lambda>0$ :

$$
(\lambda A)^{\mathrm{m} \cdot \mathrm{c} .}=\lambda(A)^{\mathrm{m} \cdot \mathrm{c} .} .
$$

We define

$$
\Sigma_{\mu g}(N, \Omega, l) \equiv N^{-1}\left(T+V_{\mu g}\right)_{\Omega}^{\text {m.c. }}
$$

and

$$
\Phi_{\mu g}(N, \beta, l) \equiv N^{-1}\left(T+V_{\mu g}\right)_{\beta}^{c}
$$

and note that the quantities of Eqs. (2.7) and (2.8) are given by

$$
\begin{aligned}
& \Sigma(N, \Omega, l)=N^{-1}\left(T+V_{0}\right)_{\Omega}^{\text {m.c. }}, \\
& \Phi(N, \beta, l)=N^{-1}\left(T+V_{0}\right)_{\beta}^{\text {c. }} .
\end{aligned}
$$

(3.13) then implies

$$
\frac{\Sigma_{\mu g}(N, \Omega, l)}{1+2 \mu^{-1 / 5}}-c \leqq \Sigma(N, \Omega, l) \leqq \frac{\Sigma_{\mu g}(N, \Omega, l)}{1-2 \mu^{-1 / 5}}+c
$$

and

$$
\Phi_{\mu g}\left(N, \frac{\beta}{1+2 \mu^{-1 / 5}}, l\right)-c \leqq \Phi(N, \beta, l) \leqq \Phi_{\mu g}\left(N, \frac{\beta}{1-2 \mu^{-1 / 5}}, l\right)+c .
$$


We have thus achieved our first goal: to replace the $1 / r$-potential by a step function with arbitrarily little effect on the thermodynamical functions.

\section{Separating the Cubes by Walls}

In this chapter it is demonstrated that separating the small cubes $\Lambda_{l / h}^{a}$ by impenetrable walls changes the specific mean (free) energy $\Sigma_{\mu g}\left(\Phi_{\mu g}\right)$ by a vanishing amount, if $N \rightarrow \infty$.

Besides the previously defined $\mathscr{G}_{N, l}$ we have to deal with another pre-Hilbert space where the wave functions vanish at the walls:

$$
\mathscr{G}_{N, l}^{W} \equiv\left\{\Phi \in \mathscr{G}_{N . l} \mid \Phi(x) \neq 0 \quad \text { implies } \quad \vec{x}_{\alpha i} \in \bigcup_{a=1}^{g} \Lambda_{l / h}^{a}(\forall \alpha, i)\right\} .
$$

(Recall that the $\Lambda_{l / h}^{a}$ are open sets.)

We shall have to compare the system without walls, volume $l^{3}$, with a system with walls, volume $l^{\prime 3}=(l+2(h-1) b)^{3} .2(h-1) b$ will eventually be chosen to be $N^{-1 / 6} l$.

$$
\begin{aligned}
\Phi, \psi \rightarrow N^{-2 / 3} & \sum_{\alpha i} \frac{1}{2 M_{\alpha}} \int d^{6 N} \boldsymbol{x}\left(\vec{\nabla}_{\alpha i} \Phi(\boldsymbol{x})\right)^{*}\left(\vec{\nabla}_{\alpha i} \psi(\boldsymbol{x})\right) \\
+ & \frac{1}{2} N^{-1} \sum_{\substack{\alpha a \\
\beta b}} W_{\alpha a, \beta b} \sum_{i, k} \int d^{6 N} \boldsymbol{x} \theta_{a}\left(\vec{x}_{\alpha i}\right) \theta_{b}\left(\vec{x}_{\beta k}\right) \Phi^{*}(\boldsymbol{x}) \psi(\boldsymbol{x})
\end{aligned}
$$

defines forms $T+V_{\mu g}: \mathscr{G}_{N, l} \times \mathscr{G}_{N, l} \rightarrow C, T^{W}+V_{\mu g}^{W}: \mathscr{G}_{N, l}^{W} \times \mathscr{G}_{N, l}^{W} \rightarrow C$ and $T^{\prime W}+V_{u a}^{\prime W}: \mathscr{G}_{N, l^{\prime}}^{W} \times \mathscr{G}_{N, l^{\prime}}^{W} \rightarrow C$. The corresponding self-adjoint operators are $\hat{T}+\hat{V}_{\mu g}: \mathscr{D}_{N, l} \rightarrow \mathscr{H}_{N, l}, \hat{T}^{W}+\hat{V}_{\mu g}^{W}: \mathscr{D}_{N, l}^{W} \rightarrow \mathscr{H}_{N, l}$ and $\hat{T}^{\prime W}+\hat{V}_{\mu g}^{\prime W}: \mathscr{D}_{N, l^{\prime}}^{W}$ $\rightarrow \mathscr{H}_{N, l^{\prime}} . T+V_{\mu g}$ describes particles which are confined to move within a cube of length $l$, each particle feels a potential which remains constant as long as it moves within the same small cube. $T^{W}+V_{\mu g}^{W}$ describes the same situation except that the particle current across the surface of any small cube vanishes. $T^{\prime W}+V_{\mu g}^{\prime W}$ describes the latter situation except that all lengths are scaled by a factor $1+2(h-1) b / l$.

Since $\mathscr{G}_{N, l}^{W} \subset \mathscr{G}_{N, l}$ we immediately obtain an estimate

and

$$
\Sigma_{\mu g}(N, \Omega, l) \leqq N^{-1}\left(T^{W}+V_{\mu g}^{W}\right)_{\Omega}^{\text {m.c. }} \equiv \sum_{\mu g}^{W}(N, \Omega, l)
$$

$$
\Phi_{\mu g}(N, \beta, l) \leqq N^{-1}\left(T+V_{\mu g}^{W}\right)_{\beta}^{\mathrm{c}} \equiv \Phi_{\mu g}^{W}(N, \beta, l) .
$$

The following discussion, the purpose of which is to obtain an estimate in the opposite direction, pertains to values of $b$ such that $4(h-1) b<l$. Since $4(h-1) b=2 N^{-1 / 6} l$ and the limit $N \rightarrow \infty$ is performed first, $g \rightarrow \infty$ 


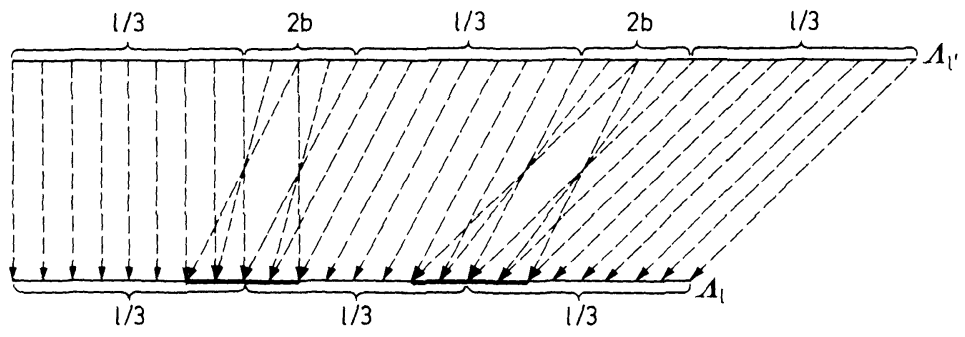

Fig. 1. The mapping $R$ from $\Lambda_{l^{\prime}}$ to $\Lambda_{l}$ for $h=3$

next, this poses no problem. Consider the (discontinuous) mapping $R: \Lambda_{l^{\prime}} \rightarrow \Lambda_{l}$ defined by:

$$
(R x)_{r} \equiv\left\{\begin{array}{l}
x_{r}-2(n-1) b \\
2 \zeta_{n}-2 n b-x_{r} \\
2 \zeta_{n}-2(n-1) b-x_{r}
\end{array}\right\} \quad \text { if }\left\{\begin{array}{l}
\zeta_{n-1}+b \leqq x_{r} \leqq \zeta_{n}-b \\
\zeta_{n}-b \leqq x_{r} \leqq \zeta_{n} \\
\zeta_{n}<x_{r} \leqq \zeta_{n}+b
\end{array}\right\}
$$

where $r=1,2,3$ labels the three spatial dimensions, $n=0,1, \ldots, h$ the walls (including those of the original cube) in a given spatial dimension, and $\zeta_{n}=n \frac{l}{h}+(2 n-1) b$ the location of such a wall.

This mapping can be depicted in a given dimension (for $h=3$ as an example) as shown in Fig. 1.

Consider next a function $f:[0, l+2(h-1) b] \rightarrow R$ with the following properties: $f$ is once continuously differentiable, $f^{2}(x) \leqq 1$; for $n=1,2, \ldots, h-1: f(x) \neq 1$ for $x \in\left[\zeta_{n}-2 b, \zeta_{n}+2 b\right]$ only; $f\left(\zeta_{n}\right)=0$; $\xi \rightarrow f\left(\zeta_{n}+\xi\right)$ is even for $|\xi| \leqq 2 b ; f^{2}\left(\zeta_{n}+\xi\right)+f^{2}\left(\zeta_{n}+2 b-\xi\right)=1$ for $0 \leqq \xi \leqq b ;\left(f^{\prime}(x)\right)^{2} \leqq{\frac{1}{b^{2}}}^{2}$. One can then show by an elementary calculation [6] that $I: \mathscr{H}_{N, l} \rightarrow \mathscr{H}_{N, l^{\prime}}$, defined by

$$
(I \Phi)\left(\vec{x}_{11}, \ldots, \vec{x}_{2 N}\right) \equiv \prod_{\alpha i} \prod_{r=1}^{3} f\left(x_{\alpha l, r}\right) \Phi\left(R \vec{x}_{11}, \ldots, R \vec{x}_{2 N}\right)
$$

is an isometry:

$$
(I \Phi, I \psi)=(\Phi, \psi) .
$$

${ }^{2}$ An example is

$$
f\left(\zeta_{n}+\xi\right)=\left\{\begin{array}{l}
\frac{1}{\sqrt{2}}\left|\frac{\xi}{b}\right|^{\sqrt{2}} \\
\sqrt{1-\frac{1}{2}\left(2-\left|\frac{\xi}{b}\right|^{2 \sqrt{2}}\right.}
\end{array}\right\} \text { if }\left\{\begin{array}{l}
|\xi| \leqq b \\
b \leqq|\xi| \leqq 2 b \\
2 b \leqq|\xi|
\end{array}\right\}
$$


Since $V_{\mu g}$ and $V_{\mu g}^{\prime W}$ are constant as long as a particle moves within a small cube one deduces as above

$$
\begin{aligned}
& \left(I \Phi\left|T^{\prime W}+V_{\mu g}^{\prime W}\right| I \Phi\right) \leqq\left(\Phi\left|T+V_{\mu g}\right| \Phi\right) \\
& \quad+\left\{\frac{3}{b^{2}} \frac{M_{1}+M_{2}}{M_{1} M_{2}} N^{1 / 3}+\frac{2(h-1) b}{l} B N\right\}(\Phi, \Phi)
\end{aligned}
$$

holds for $\Phi \in \mathscr{G}_{N, l}$ where $B=\frac{1}{2} \sum_{\substack{\alpha a \\ \beta b}}\left|W_{\alpha a^{\prime} \beta b}\right|$ does not depend on $N$. The

first term in curly brackets estimates the change in kinetic energy, the second takes into account the $1^{-1}$-factor in (3.5). This demonstrates in particular that $I \mathscr{G}_{N, l} \subset \mathscr{G}_{N, l^{\prime}}^{W}$. (Recall that the conditions on $f$ imply $f^{\prime}\left(\zeta_{n}\right)=0$.) Therefore, if $\mathscr{F}=\left\{\Phi_{1}, \Phi_{2}, \ldots\right\}$ is a set of normalized and mutually orthogonal vectors in the domain of the form $T+V_{\mu g}$ the set $I \mathscr{F}=\left\{I \Phi_{1}, I \Phi_{2}, \ldots\right\}$ is an orthonormal set of vectors in the domain of $T^{\prime W}+V_{\mu g}^{\prime W}$, from which property the estimates

$$
\begin{aligned}
& \Sigma_{\mu g}^{W}\left(N, \Omega, l+N^{-1 / 6} l\right) \leqq \Sigma_{\mu g}(N, \Omega, l)+d, \\
& \Phi_{\mu g}^{W}\left(N, \Omega, l+N^{-1 / 6} l\right) \leqq \Phi_{\mu g}(N, \Omega, l)+d
\end{aligned}
$$

follow for $N>2^{6}$ with $d=\frac{12 g^{2 / 3}}{l^{2}}\left(\frac{1}{M_{1}}+\frac{1}{M_{2}}\right) N^{-1 / 3}+B N^{-1 / 6}$.

With (4.3), (4.4), (4.9), and (4.10) we have reached the goal of this chapter.

\section{Distributing Particles Among Cubes}

We now want to show why, in the thermodynamic limit, just one particular distribution of particles among cubes $\Lambda_{l / h}^{a}$ is all we have to take into account. For the canonical case this will be obvious, but for the microcanonical mean energy of the first $\Omega$ levels we need a somewhat painful analysis.

First of all it is clear that one obtains a higher mean energy by taking all $\Omega$ eigenvalues for one particle distribution than minimizing over all possibilities. However, since $\Omega$ is much larger than the (finite) number of distributions the absolute minimum is larger than the one for a fixed distribution and $\Omega$ divided by the number of distributions. But in the limit $N \rightarrow \infty$ for $s=\frac{1}{N} \ln \Omega$ such a factor does not matter.

To sharpen these ideas denote by $0<\varepsilon_{1} \leqq \varepsilon_{2} \leqq \cdots$ the points of the (discrete) spectrum of the kinetic energy operator $\hat{t}^{(1)}$ describing one particle in a cube $0 \leqq x_{r} \leqq \frac{l}{h}(r=1,2,3)$. 
We define the following sets of partitions (with $\alpha=1,2 ; a=1,2, \ldots, g$; $j=1,2, \ldots)$ :

with

$$
X \equiv\left\{v \mid v_{\alpha a j}=0,1, \sum_{a j} v_{\alpha a j}=N\right\}
$$

$$
\left[X^{\prime}\right]_{\Omega^{\prime}} \equiv\left\{X^{\prime \prime} \mid X^{\prime \prime} \subset X^{\prime} \subset X, X^{\prime \prime} \text { contains } \Omega^{\prime} \text { elements }\right\}
$$

for $\Omega^{\prime} \in \mathbb{N}$,

$$
\begin{gathered}
Y \equiv\left\{n \mid n_{\alpha a} \in \mathbb{N}, \sum_{a} n_{\alpha a}=N\right\}, \\
X_{n} \equiv\left\{v \in X \mid \sum_{j} v_{\alpha a j}=n_{\alpha a}\right\} \text { for } n \in Y,
\end{gathered}
$$

and

$$
\mathcal{O} \equiv\left\{\omega \mid \omega_{n} \in \mathbb{N}(n \in Y), \sum_{n \in Y} \omega_{n}=\Omega\right\}
$$

Note that $\mathcal{O}$ and $Y$ are finite sets; $\sum_{n \in Y}=\left(\begin{array}{c}N+g-1 \\ g-1\end{array}\right)^{2}$. Each partition $v \in X$ corresponds to precisely one eigenvalue of $\hat{T}^{W}+\hat{V}_{\mu g}^{W}$ of the preceding chapter, namely to

$$
E_{v}=N^{-2 / 3} \sum_{\alpha a j} v_{\alpha a j} \frac{\varepsilon_{j}}{2 M_{\alpha}}+\frac{1}{2} N^{-1} \sum_{\substack{\alpha a j \\ \beta b k}} v_{\alpha a j} W_{\alpha a, \beta b} v_{\beta b k} .
$$

Note that Fermi-Dirac statistics has been taken into account by allowing $v_{\alpha a j}=0$ or 1 only.

The quantities under discussion here are

and

$$
\Sigma_{\mu g}^{W}(N, \Omega, l)=\frac{1}{N \Omega} \inf _{X^{\prime} \in[X]_{\Omega}} \sum_{v \in X^{\prime}} E_{v}
$$

$$
\Phi_{\mu g}^{W}(N, \beta, l)=-\frac{1}{\beta N} \log \sum_{v \in X} e^{-\beta E_{v}} .
$$

They will be replaced by

$$
\sum_{\mu g}^{M}(N, \Omega, l) \equiv \min _{n \in Y} \inf _{X^{\prime} \in\left[X_{n}\right]_{\Omega}} \frac{1}{N \Omega} \sum_{v \in X^{\prime}} E_{v}
$$

and

$$
\Phi_{\mu g}^{M}(N, \beta, l) \equiv \min _{n \in Y}\left\{-\frac{1}{\beta N} \log \sum_{\nu \in X_{n}} e^{-\beta E_{\nu}}\right\},
$$

respectively.

From

$$
e^{-\beta N \Phi_{M g}^{M}(N, \beta, l)} \leqq \sum_{n \in Y} \sum_{\nu \in X_{n}} e^{-\beta E_{v}} \leqq e^{-\beta N \Phi_{\mu g}^{M}(N, \beta, l)} \sum_{n \in Y}
$$


we deduce immediately

$\Phi_{\mu g}^{M}(N, \beta, l) \geqq \Phi_{\mu g}^{W}(N, \beta, l) \geqq \Phi_{\mu g}^{M}(N, \beta, l)-\frac{1}{\beta N} \log \left(\begin{array}{c}N+g-1 \\ g-1\end{array}\right)^{2}$.

If, in the case of the microcanonical ensemble the minimum over $\omega \in \mathcal{O}$ in

$$
\inf _{X^{\prime} \in[X]_{\Omega}} \sum_{v \in X^{\prime}} E_{v}=\min _{\omega \in \mathcal{O}} \sum_{n \in Y^{\prime}} \inf _{X^{\prime \prime} \in\left[X_{n}\right]_{\omega_{n}}} \sum_{v \in X^{\prime \prime}} E_{v}
$$

is restricted to those $\omega$ where $\omega_{n}$ vanishes for all but one $n \in Y$, the estimate

$$
\Sigma_{\mu g}^{W}(N, \Omega, l) \leqq \Sigma_{\mu g}^{M}(N, \Omega, l)
$$

follows immediately.

On the other hand, the minimum over $\omega \in \mathcal{O}$ in (5.12) is attained for a particular $\omega^{0} \in \mathcal{O}$. Denote by $Y_{0}$ the set of those particle number distributions $m \in Y$ for which

$$
\omega_{m}^{0}<\Omega^{\prime} \equiv \frac{\Omega}{N \sum_{n \in Y}}
$$

is satisfied. Note that $0 \leqq \delta \equiv \sum_{n \in Y_{0}} \frac{\omega_{n}^{0}}{\Omega} \leqq \frac{1}{N}$ is a consequence. The sum over $n \in Y$ in (5.12) splits into two parts:

$$
\frac{1}{N \Omega} \sum_{n \in Y_{0}} \inf _{X^{\prime} \in\left[X_{n}\right\rfloor_{\omega_{n}^{0}}} \sum_{v \in X^{\prime}} E_{v} \geqq \sum_{n \in Y_{0}} \frac{\omega_{n}^{0}}{\Omega} \inf _{v \in X} \frac{E_{v}}{N} \geqq-\frac{1}{N} B
$$

- with $B=\frac{1}{2} \sum_{\substack{\alpha a \\ \beta b}}\left|W_{\alpha a, \beta b}\right|$ as in Chapter IV - and a sum over $n \in Y-Y_{0}$. Now, since $\Omega^{\prime \prime} \rightarrow \inf _{X^{\prime} \in\left[X_{n}\right] \Omega_{2^{\prime \prime}}} \frac{1}{\Omega^{\prime \prime}} \sum_{\nu \in X^{\prime}} N^{+2 / 3} \sum_{\alpha a j} \frac{v_{\alpha a j} \varepsilon_{j}}{2 M_{\alpha}}$ increases, so does $\Omega^{\prime \prime} \rightarrow \inf _{X^{\prime} \in\left[X_{n}\right] \Omega^{\prime \prime}} \frac{1}{\Omega^{\prime \prime}} \sum_{v \in X^{\prime}} E_{v}$ for fixed $n$. Consequently

$$
\begin{aligned}
(1-\delta) \Sigma_{\mu g}^{M}\left(N, \Omega^{\prime}, l\right) & \leqq \sum_{n \in Y-Y_{0}} \frac{\omega_{n}^{0}}{\Omega} \inf _{X^{\prime} \in\left[X_{n}\right] \Omega^{\prime}} \frac{1}{N \Omega^{\prime}} \sum_{v \in X^{\prime}} E_{v} \\
& \leqq \sum_{n \in Y-Y_{0}} \frac{\omega_{n}^{0}}{\Omega} \inf _{X^{\prime} \in\left[X_{n}\right] \omega_{n}^{\circ}} \frac{1}{N \omega_{n}^{0}} \sum_{v \in X^{\prime}} E_{v},
\end{aligned}
$$

so that

$$
(1-\delta) \Sigma_{\mu g}^{M}\left(N, \Omega^{\prime}, l\right)-\frac{B}{N} \leqq \Sigma_{\mu g}^{W}(N, \Omega, l)
$$

has been established. 
Recall that $B$ does not depend on $N, 0 \leqq \delta \leqq \frac{1}{N}$. Since

$$
N\left(\begin{array}{c}
N+g-1 \\
g-1
\end{array}\right)^{2} \Omega^{\prime}=\Omega
$$

we have $\lim _{N \rightarrow \infty} \frac{1}{N} \log \Omega=\lim _{N \rightarrow \infty} \frac{1}{N} \log \Omega^{\prime}$. (5.11), (5.13) and (5.16) say that, in the limit $N \rightarrow \infty$, all but one particle distributions $n \in Y$ give negligible contributions.

\section{The Thomas-Fermi Equation}

Consider sequences $\Omega_{N}, \beta_{N}, l_{N}$ with $N^{-1} \log \Omega_{N} \rightarrow s>0, \beta_{N} \rightarrow \beta>0$ and $l_{N} \rightarrow l>0$. In this chapter we shall derive an explicit expression for

and

$$
\Sigma_{\mu g}(s, l) \equiv \lim _{N \rightarrow \infty} \Sigma_{\mu g}^{M}\left(N, \Omega_{N}, l_{N}\right)
$$

$$
\Phi_{\mu g}(\beta, l) \equiv \lim _{N \rightarrow \infty} \Phi_{\mu g}^{M}\left(N, \beta_{N}, l_{N}\right)
$$

The problem now is to interchange this limit with taking the minimum by which $\Sigma^{M}$ and $\Phi^{M}$ are defined. For this purpose we introduce the fraction $z_{\alpha a}=n_{\alpha a} / N$ of particles of species $\alpha$ in cube $a$. In other words, we rewrite the minimum over particle distributions as a minimum over the simplex

$$
Z_{N} \equiv\left\{z \mid N z_{\alpha a} \in \mathbb{N}, \sum_{a} z_{\alpha a}=1\right\}
$$

and, in the limit $N \rightarrow \infty$, over

$$
Z \equiv \overline{\bigcup_{N \in \mathbb{N}} Z_{N}}=\left\{z \mid z_{\alpha a} \geqq 0, \sum_{a} z_{\alpha a}=1\right\} .
$$

For a particular $z \in Z_{N}$ with corresponding $n=N z \in Y$ we define

$$
\begin{gathered}
\varepsilon_{g}(N, \Omega, l, z) \equiv \inf _{X^{\prime} \in\left[X_{n}\right] \Omega} \frac{1}{N \Omega} \sum_{\nu \in X_{n}} N^{-2 / 3} \sum_{\alpha a j} \frac{v_{\alpha a j} \varepsilon_{j}}{2 M_{\alpha}}, \\
\phi_{g}(N, \beta, l, z) \equiv-\frac{1}{\beta N} \log \sum_{\nu \in X_{n}} e^{-\beta N^{-2 / 3} \sum_{\alpha a j} \frac{v_{\alpha a j} \varepsilon_{j}}{2 M_{\alpha}}}
\end{gathered}
$$

and

$$
u_{\mu g}(z) \equiv \frac{1}{2} \sum_{\substack{\alpha a \\ \beta b}} z_{\alpha a} W_{\alpha a, \beta b} z_{\beta b} .
$$


Recall that the dependence of $\varepsilon$ and $\phi$ on $l$ results from the dependence on $l$ of the eigenvalues $\varepsilon_{j}$ of $\hat{t}^{(1)} \cdot \varepsilon_{g}(N, \Omega, l, z)\left(\phi_{g}(N, \beta, l, z)\right)$ is the mean (free) energy per particle pair for two species of noninteracting fermions ( $N$ each), with entropy $\log \Omega$ (temperature $\beta^{-1}$ ), if the containing volume $N l^{3}$ is subdivided into $g$ equal cubes and if there are $N z_{\alpha a}$ particles of kind $\alpha$ in cube $a$.

If $z_{N} \in Z_{N}$ is a sequence of particle distributions which converges towards $z \in Z$ (component-wise), the limits

$$
\begin{gathered}
\varepsilon_{g}(s, l, z) \equiv \lim _{N \rightarrow \infty} \varepsilon_{g}\left(N, \Omega_{N}, l_{N}, z_{N}\right), \\
\phi_{g}(\beta, l, z) \equiv \lim _{N \rightarrow \infty} \phi_{g}\left(N, \beta_{N}, l_{N}, z_{N}\right)
\end{gathered}
$$

are known to exist [7] and are given by the Legendre-transforms of the grand-canonical ensemble expression:

$$
\varepsilon_{g}(s, l, z)=\frac{l^{3}}{g} \sum_{\alpha a} \int \frac{d^{3} q}{(2 \pi)^{3}} \frac{q^{2}}{2 M_{\alpha}} \frac{1}{1+e^{\beta \frac{q^{2}}{2 M_{\alpha}}-\beta \mu_{\alpha a}}},
$$

with $\beta, \mu_{\alpha a}$ as unique solutions of

and

$$
z_{\alpha a}=\frac{l^{3}}{g} \int \frac{d^{3} q}{(2 \pi)^{3}} \frac{1}{1+e^{\beta \frac{q^{2}}{2 M_{\alpha}}-\beta \mu_{\alpha a}}}
$$

$$
\begin{aligned}
s=\sum_{\alpha a}\left\{-\beta z_{\alpha a} \mu_{\alpha a}+\beta \frac{l^{3}}{g} \int\right. & \frac{d^{3} q}{(2 \pi)^{3}} \frac{q^{2}}{2 M_{\alpha}} \frac{1}{1+e^{\beta \frac{q^{2}}{2 M_{x}}-\beta \mu_{x a}}} \\
+ & \left.\frac{l^{3}}{g} \int \frac{d^{3} q}{(2 \pi)^{3}} \log \left(1+e^{-\beta \frac{q^{2}}{2 M_{x}}+\beta \mu_{x a}}\right)\right\} .
\end{aligned}
$$

Likewise:

$$
\phi_{g}(s, l, z)=\sum_{\alpha a}\left\{z_{\alpha a} \mu_{\alpha a}-\frac{1}{\beta} \frac{l^{3}}{g} \int \frac{d^{3} q}{(2 \pi)^{3}} \log \left(1+e^{-\beta \frac{q^{2}}{2 M_{\alpha}}+\beta \mu_{\alpha a}}\right)\right\}
$$

where the $\mu_{\alpha a}$ are to be determined from

$$
z_{\alpha a}=\frac{l^{3}}{g} \int \frac{d^{3} q}{(2 \pi)^{3}}-\frac{1}{1+e^{\beta \frac{q^{2}}{2 M_{\alpha}}-\beta \mu_{\alpha a}}}
$$

With definitions (6 1), (6.2), (6.5), (6.6), and (6.7) we can rewrite (5.8) and (5.9):

$$
\begin{gathered}
\Sigma_{\mu g}(s, l)=\lim _{N \rightarrow \infty} \min _{z \in Z_{N}}\left\{u_{\mu g}(z)+\varepsilon_{g}\left(N, \Omega_{N}, l_{N}, z\right)\right\}, \\
\Phi_{\mu g}(\beta, l)=\lim _{N \rightarrow \infty} \min _{z \in Z_{N}}\left\{u_{\mu g}(z)+\phi_{g}\left(N, \beta_{N}, l_{N}, z\right)\right\} .
\end{gathered}
$$


Let us abbreviate the expression in curly bracket in (6.15) or (6.16) by $\chi_{N}(z)$. Since $z \rightarrow u_{\mu g}(z)$ is continuous we conclude with the remarks before (6.8) and (6.9) that $\chi_{N}\left(z_{N}\right)$ converges towards $\chi(z)$ for any sequence $z_{N} \in Z_{N}$ which converges towards a $z \in Z$. An inspection of (6.7), (6.10) to (6.14) shows that $z \rightarrow \chi(z)$ is continuous and hence $\inf _{z \in Z} \chi(z)$ is attained for a certain $\bar{z}$. If $z_{N} \in Z_{N}$ is a sequence which converges towards $\bar{z}$ we conclude:

$$
\inf _{z \in Z} \chi(z)=\lim _{N \rightarrow \infty} \chi_{N}\left(z_{N}\right) \geqq \limsup _{N \rightarrow \infty} \min _{z \in Z_{N}} \chi_{N}(z) .
$$

On the other hand, there is a sequence $\bar{z}_{N}$ defined by $\min _{z \in Z_{N}} \chi_{N}\left(z_{N}\right)=\chi_{N}\left(\bar{z}_{N}\right)$. Since $Z$ is compact there exists an ascending sequence $N_{i}$ of integers such that $\bar{z}_{N_{i}}$ converges with $i \rightarrow \infty$. Consequently $\lim _{i \rightarrow \infty} \chi_{N_{i}}\left(\bar{z}_{N_{i}}\right)=\chi\left(\lim _{i \rightarrow \infty} \bar{z}_{N_{i}}\right)$ $\geqq \inf _{z \in Z} \chi(z)$, and therefore

$$
\liminf _{N \rightarrow \infty} \min _{z \in Z_{N}} \chi_{N}(z) \geqq \inf _{z \in Z} \chi(z) .
$$

(6.17) and (6.18) together imply

$$
\lim _{N \rightarrow \infty} \min _{z \in Z_{N}} \chi_{N}(z)=\min _{z \in Z} \lim _{N \rightarrow \infty} \chi_{N}(z) .
$$

We have thus established:

$$
\Sigma_{\mu g}(s, l)=\inf _{z \in Z}\left\{u_{\mu g}(z)+\varepsilon_{g}(s, l, z)\right\}
$$

and

$$
\Phi_{\mu g}(\beta, l)=\inf _{z \in Z}\left\{u_{\mu g}(z)+\phi_{g}(\beta, l, z)\right\} .
$$

The particle distribution $\bar{z}$ where the infimum of (6.20) or (6.21) is attained is not a point on the boundary of $Z^{3}$. Consequently the variation of the expressions in curly brackets in (6.20) or (6.21) must vanish somewhere in $Z$.

Because of (6.11) and (6.12) we have

$$
\beta^{-1} \delta s=\delta \varepsilon_{g}-\sum_{\alpha a} \mu_{\alpha a} \delta z_{\alpha a},
$$

so that for a variation of $\Sigma$ at constant $s$ we calculate

$$
0=\sum_{\alpha a}\left\{\sum_{\beta b} W_{\alpha a, \beta b} \bar{z}_{\beta b}+\bar{\mu}_{\alpha a}\right\} \delta z_{\alpha a}, \sum_{a} \delta z_{\alpha a}=0
$$

${ }^{3}$ The functions on the right hand side of (6.20) and (6.21) are explicitly known and are seen to be differentiable in the interior of $Z$, the partial derivative with respect to $z_{x a}$ at the boundary turns out to be positive infinite. 
with $\bar{\mu}_{\alpha a}$ the local chemical potentials corresponding to $\bar{z}_{\alpha a}$. The solution of $(6.23)$ is

$$
\bar{\mu}_{\alpha a}=\mu_{\alpha}-\sum_{\beta b} W_{\alpha a, \beta b} \bar{z}_{\beta b} .
$$

The minimizing particle distribution is a solution of (6.11) with (6.24):

$$
\bar{z}_{\alpha a}=\frac{l^{3}}{g} \int \frac{d^{3} q}{(2 \pi)^{3}} \frac{1}{\left.1+e^{\beta\left[\frac{q^{2}}{2 M_{\alpha}}\right.}+\sum_{\beta b} W_{\alpha a, \beta b} \bar{z}_{\beta b}-\mu_{\alpha}\right]},
$$

where $\mu_{1}, \mu_{2}$ and $\beta$ are to be determined from

$$
\begin{gathered}
\sum_{a} \bar{z}_{\alpha a}=1 \quad(\alpha=1,2), \\
s=2 \beta u_{\mu g}(\bar{z})-\beta\left(\mu_{1}+\mu_{2}\right) \\
\left.+\frac{l^{3}}{g} \sum_{\alpha a} \int \frac{d^{3} q}{(2 \pi)^{3}} \log \left(1+e^{-\beta\left[\frac{q^{2}}{2 M_{\alpha}}\right.}+\sum_{\beta b} W_{\alpha a, \beta b} \bar{z}_{\beta b}-\mu_{\alpha}\right]\right) \\
+\beta \frac{l^{3}}{g} \sum_{\alpha a} \int \frac{d^{3} q}{(2 \pi)^{3}} \frac{q^{2}}{2 M_{\alpha}} \frac{1}{1+e^{\beta\left[\frac{q^{2}}{2 M_{\alpha}}+\sum_{\beta b} W_{\alpha a, \beta b} \bar{z}_{\beta b}-\mu_{\alpha}\right]}} .
\end{gathered}
$$

If this system of Eqs. (6.25) to (6.27) should allow more than one solutionthe foregoing discussion has shown that there is at least one - we must choose that for which

$$
u_{\mu g}(\bar{z})+\frac{l^{3}}{g} \sum_{\alpha a} \int \frac{d^{3} q}{(2 \pi)^{3}} \frac{q^{2}}{2 M_{\alpha}} \frac{1}{1+e^{\beta\left[\frac{q^{2}}{2 M_{\alpha}}+\sum_{\beta b} W_{\alpha a, \beta b} \bar{z}_{\beta b}-\mu_{\alpha}\right]}}
$$

is a minimum. The minimal expression (6.28) coincides with $\Sigma_{\mu g}(s, l)$.

In the case of the canonical ensemble the same analysis yields: The minimizing particle distribution $\bar{z}$ is a solution of (6.25) and (6.26). This system of equations has at least one solution, if there are many solutions we have to choose that for which$$
-\bar{u}_{\mu g}(\bar{z})+\mu_{1}+\mu_{2}-\frac{1}{\beta} \frac{l^{3}}{g} \sum_{\alpha a} \int \frac{d^{3} q}{(2 \pi)^{3}} \log \left(1+e^{-\beta\left[\frac{q^{2}}{2 M_{x}}+\sum_{\beta b} W_{\alpha a, \beta b} \bar{z}_{\beta b}-\mu_{x}\right]}\right)
$$

is a minimum. The minimal expression (6.29) coincides with $\Phi_{\mu g}(\beta, l)$.

The system of Eqs. (6.25) to (6.28) is the Thomas-Fermi equation in the case of the microcanonical ensemble, (6.25), (6.26), and (6.29) in the case of the canonical ensemble. 


\section{The Final Result}

We shall now put together the estimates of Chapters III to V and combine them with the explicit expressions for (6.1) and (6.2). Note that (6.1) and (6.2) contain arbitrary converging sequences $N^{-1} \log \Omega_{N}, \beta_{N}$, and $l_{N}$. With definitions (2.9) and estimates (3.23), (4.3), (4.9), (5.13), and (5.16) we conclude:

$$
\Sigma(s, l)=\lim _{\mu \rightarrow \infty} \lim _{g \rightarrow \infty} \Sigma_{\mu g}(s, l),
$$

from (2.10), (3.24), (4.4), (4.10), and (5.11)

$$
\Phi(\beta, l)=\lim _{\mu \rightarrow \infty} \lim _{g \rightarrow \infty} \Phi_{\mu g}(\beta, l) .
$$

The expressions on the right hand side are to be calculated by solving the corresponding Thomas-Fermi equation. We shall not demonstrate in this paper that the limits $g \rightarrow \infty, \mu \rightarrow \infty$ on the right hand sides of Eqs. (7.1) and (7.2) exist and are to be calculated by solving the ThomasFermi equation with $v\left(\vec{x}^{\prime}, \vec{x}^{\prime \prime}\right)=\left|\vec{x}^{\prime}-\vec{x}^{\prime \prime}\right|^{-1}$ instead of

$$
v_{\mu g}\left(\vec{x}^{\prime}, \vec{x}^{\prime \prime}\right)=\sum_{a b} \theta_{a}\left(\vec{x}^{\prime}\right) \frac{1-e^{-\mu\left|\vec{\xi}_{a}-\vec{\xi}_{b}\right|}}{\left|\vec{\xi}_{a}-\vec{\xi}_{b}\right| l} \theta_{b}\left(\vec{x}^{\prime \prime}\right) .
$$

We refer the reader to the proof given in the appendix of an earlier paper [3]. We shall state the result only:

For given $\beta>0, l>0$ there are solutions of the Thomas-Fermi equation

with

$$
\varrho_{\alpha}(\vec{x})=\int \frac{d^{3} q}{(2 \pi)^{3}} \frac{1}{1+e^{\beta\left[\frac{q^{2}}{2 M_{x}}+W_{\alpha}(\vec{x})-\mu_{\alpha}\right]}}
$$

$$
W_{\alpha}(\vec{x})=\sum_{\beta} \int d^{3} x^{\prime} \frac{e_{\alpha} e_{\beta}-\kappa M_{\alpha} M_{\beta}}{\left|\vec{x}-\vec{x}^{\prime}\right|} \varrho_{\beta}\left(\vec{x}^{\prime}\right),
$$

which are normalized

$$
\int_{\Lambda_{l}} d^{3} x \varrho_{\alpha}(\vec{x})=1 \quad(\alpha=1,2) .
$$

One then calculates

$$
u=\sum_{\alpha} \int_{\Lambda_{l}} d^{3} x \varrho_{\alpha}(\vec{x}) W_{\alpha}(\vec{x}),
$$

$$
\begin{aligned}
& \phi=-u+\sum_{\alpha}\left\{\mu_{\alpha}-\frac{1}{\beta} \int_{\Lambda_{l}} d^{3} x \int \frac{d^{3} q}{(2 \pi)^{3}} \log \left(1+e^{-\beta\left[\frac{q^{2}}{2 M_{\alpha}}+W_{\alpha}(\vec{x})-\mu_{\alpha}\right]}\right)\right\}, \\
& \text { and } \\
& \varepsilon=u+\sum_{\alpha} \int_{\Lambda_{l}} d^{3} x \int \frac{d^{3} q}{(2 \pi)^{3}} \frac{q^{2}}{2 M_{\alpha}} \frac{1}{1+e^{\beta\left[\frac{q^{2}}{2 M_{\alpha}}+W_{\alpha}(\vec{x})-\mu_{\alpha}\right]}},
\end{aligned}
$$

$$
s=\beta(\varepsilon-\phi) .
$$


If (7.3) to (7.5) has more than one solution that which minimizes $\phi$ has to be chosen, and the minimal $\phi$ coincides with $\Phi(\beta, l)$. From $\beta \rightarrow s$, $\beta \rightarrow \varepsilon$ the graph $\varepsilon(s, l)$ is constructed by choosing the smaller one of two possible $\varepsilon$ 's. $\varepsilon(s, l)$ coincides with $\Sigma(s, l)$. From (6.22) and (7.9) it follows that the microcanonical and the canonical thermodynamical functions are Legendre-transforms of each other if the Legendre-transform is possible, i.e. for those values of the entropy $s$ where

$$
\frac{\partial^{2} \Sigma(s, l)}{\partial s^{2}}>0
$$

\section{References}

1. Hertel, P., Thirring, W.: Commun. math. Phys. 24, 22 (1971).

2. Lévy-Leblond, J. M.: J. Math. Phys. 10, 806 (1969).

3. Hertel, P., Thirring, W.: Thermodynamic instability of a system of gravitating fermions. In: Dürr, H.P. (Ed.): Quanten und Felder. Braunschweig: Vieweg 1971.

4. Aronson, E, B., Hansen, C.J.: Thermal equilibrium states of a classical system with gravitation. University of Colorado preprint (1972).

5. Carlitz, R.D.: Hadronic matter at high density. Institute for Advanced Studies preprint (1972).

6. Robinson,D.W.: The Thermodynamic Pressure in Statistical Mechanics. Springer lecture notes (1971).

7. Ruelle, D.: Statistical mechanics - Rigorous Results. New York-Amsterdam: W. A. Benjamin 1969.

P. Hertel

H. Narnhofer

W. Thirring

Institute for Theoretical Physics

University of Vienna

A-1090 Vienna, Austria 